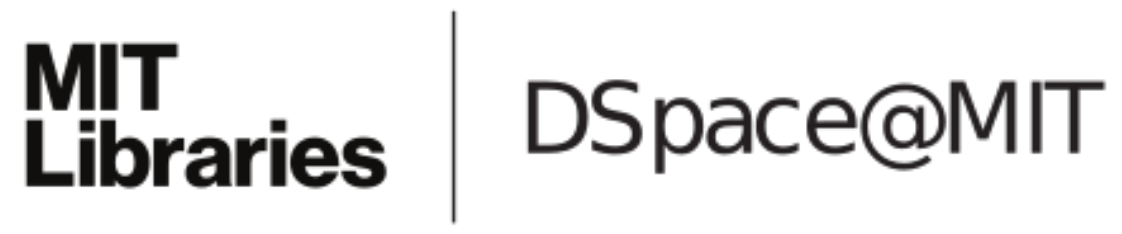

\author{
MIT Open Access Articles
}

Measurement of fast electron transport by lower hybrid modulation experiments in Alcator C-Mod

The MIT Faculty has made this article openly available. Please share how this access benefits you. Your story matters.

Citation: Schmidt, A. E. et al. "Measurement of Fast Electron Transport by Lower Hybrid Modulation Experiments in Alcator C-Mod." AIP Conf. Proc., November 26, 2009, Volume 1187, pp. 339-342.

As Published: http://dx.doi.org/10.1063/1.3273762

Publisher: American Institute of Physics

Persistent URL: http://hdl.handle.net/1721.1/66991

Version: Author's final manuscript: final author's manuscript post peer review, without publisher's formatting or copy editing

Terms of use: Creative Commons Attribution-Noncommercial-Share Alike 3.0 


\title{
Measurement of Fast Electron Transport by Lower Hybrid Modulation Experiments in Alcator C-Mod
}

\author{
A. E. Schmidt ${ }^{\mathrm{a}}$, P. T. Bonoli ${ }^{\mathrm{a}}$, R. Parker ${ }^{\mathrm{a}}$, M. Porkolab ${ }^{\mathrm{a}}$, G. Wallace, \\ J. C. Wright ${ }^{\mathrm{a}}$, J. R. Wilson ${ }^{\mathrm{b}}$, R. W. Harvey ${ }^{\mathrm{c}}$, and A. P. Smirnov ${ }^{\mathrm{c}}$ \\ ${ }^{a}$ MIT Plasma Science and Fusion Center, Cambridge, Massachusetts, USA \\ ${ }^{b}$ Princeton Plasma Physics Laboratory, Princeton, New Jersey, USA \\ ${ }^{c}$ CompX Corporation, Del Mar, California, USA
}

\begin{abstract}
The Lower Hybrid Current Drive (LHCD) system on Alcator C-Mod can produce spectra with a wide range of peak parallel refractive index $\left(\mathrm{n}_{\|}\right)$. An experiment in which LH power is square-wave modulated on a time scale much faster than the current relaxation time does not significantly alter the poloidal magnetic field inside the plasma and thus allows for realistic modeling and consistent plasma conditions for different $\mathrm{n}_{\|}$spectra. Boxcar binning of hard x-rays during LH power modulation allows for time resolution sufficient to resolve the build-up, steady-state, and slowing-down of fast electrons. A transport model built in Matlab has been used to determine a fast electron pinch velocity for a high- $\mathrm{n}_{\|}$case of 1-2 m/s.
\end{abstract}

Keywords: lower hybrid current drive, fast electrons, diffusion, pinch, power modulation, Bremsstrahlung, hard x-rays, transport

PACS: 52.35.Hr, 52.55.Fa, 52.55.Wq, 52.70.La

\section{LOWER HYBRID POWER MODULATION EXPERIMENTS}

In order to control the location of LH-driven current, it is important to understand where in the plasma the LH power is deposited as well as the transport properties of the current-carrying fast electrons. To this end, there have been several studies of fast electron diffusion in LHCD tokamak plasmas ${ }^{1,2}$ which have determined an upper bound on fast electron diffusion in the range of $0.2-2 \mathrm{~m}^{2} / \mathrm{s}$. Recent experiments on Alcator C-Mod have revealed the build-up of a radial electric field in the core of the plasma during LHCD operation, ${ }^{3,4}$ speculated to be caused by an inward pinch of LHdriven fast electrons.

In this experiment, designed to measure transport properties of the LH-driven fast electrons, LH power was square-wave modulated with a $25 \mathrm{~ms}$ period and a $50 \%$ duty cycle. Bremsstralung radiation from the resulting fast electrons was measured with Alcator C-Mod's 32 spatial chord hard x-ray (HXR) camera. ${ }^{5}$ The camera's pulse trains are digitized and post-processed for flexible time and energy binning. The resulting HXR emission was boxcar binned in time for high signal-to-noise, high timeresolution x-ray time traces. This signal was Fourier decomposed, spatially inverted, and fit to a transport model to determine the magnitude of fast electron pinch velocity. Peak coupled LH power was approximately $400 \mathrm{~kW}$. In the case analyzed in this paper, $\mathrm{n}_{\|}=3.1, \mathrm{n}_{\mathrm{e}}=8.3 \times 10^{19} \mathrm{~m}^{-3}, \mathrm{~T}_{\mathrm{e} 0}=2.3 \mathrm{keV}$, and $\mathrm{I}_{\mathrm{p}}=800 \mathrm{kA}$. 


\section{TRANSPORT FORMULATION}

The fast electron diffusion coefficients and pinch velocities can be determined with a model that fits various terms to the real and imaginary Fourier components of the HXR data. The data is Fourier decomposed and then spatially inverted to be a function of radius.

First, we assume that the HXR emission is proportional to the product of the bulk electron density and the fast electron number density. The fast electron number density, which will be referred to as $\mathrm{n}(\mathrm{r}, \mathrm{t})$, should obey the simplified transport equation, Eq. 1. In Eq. 1, $\tau$ is a fast electron slowing-down time, D is a diffusion coefficient, $v$ is a radial velocity, and $S(r, t)$ is a source term of fast electrons. This analysis is done in cylindrical geometry, with toroidal and non-circular effects ignored. The coordinate $\mathrm{r}$ refers to normalized radius, $\mathrm{r} / \mathrm{a}$.

$$
\frac{\partial n}{\partial t}=-\frac{n}{\tau}-\nabla \cdot(n \boldsymbol{v})+\nabla \cdot(D \nabla n)+S
$$

We can Fourier decompose $\mathrm{n}(\mathrm{r}, \mathrm{t})$, as in Eq. 2. We assume that the radial dependence of $\tau$ is proportional to the inverse of the bulk electron density profile. In the local region where we will be doing the analysis, we assume constant $\mathrm{v}$ and $\mathrm{D}$. Assuming that the $\mathrm{S}(\mathrm{r}, \mathrm{t})$ term is proportional to the LH power trace, it is a square wave in time with an unknown dependence on radius. In this model, we have assumed a parabolic radial dependence of $\mathrm{S}(\mathrm{r}, \mathrm{t})$ in a local region of the plasma where HXR emission is peaked, a reasonable assumption that is consistent with full-wave LH code results $^{6}$. A small time base difference between the $\mathrm{LH}$ power trace and the HXR signal, $\delta$ t, is included in the model. Substituting Eq. 2 into Eq. 1, adding the above assumptions, and collecting sine and cosine terms, yields Eq. 3 and Eq. 4 for every harmonic $l$.

$$
\begin{gathered}
n(r, t)=n_{0}(r, t)+n_{1 i} \sin (\omega t)+n_{1 r} \cos (\omega t)+n_{3 i} \sin (3 \omega t)+n_{3 r} \cos (3 \omega t)+\ldots \\
l \omega n_{l i}(r)=-\frac{n_{l r}(r) n_{e}(r)}{\tau_{0} n_{0}}-v \nabla \cdot\left(n_{l r}(r) \hat{\boldsymbol{r}}\right)+D \nabla^{2} n_{l r}(r)-\frac{\sin (l \omega \delta t)}{l}\left[S_{\max }-\frac{\left(r-r_{\max }\right)^{2}}{r_{\text {width }}}\right] \\
l \omega n_{l r}(r)=\frac{n_{l i}(r) n_{e}(r)}{\tau_{0} n_{0}}+v \nabla \cdot\left(n_{l i}(r) \hat{\boldsymbol{r}}\right)-D \nabla^{2} n_{l i}(r)-\frac{\cos (l \omega \delta t)}{l}\left[S_{\max }-\frac{\left(r-r_{\max }\right)^{2}}{r_{\text {width }}}\right]
\end{gathered}
$$

The analysis technique used to solve for transport coefficients and source term parameters has been verified by a simple PDE solver that evolves a density profile in time, given a source term, a diffusion coefficient, a pinch velocity, and a slowingdown time. The resulting time-dependent fast electron density profile can be run through the analysis technique described in this paper. A wide range of input parameters as well as different source profile shapes (parabolic, Lorentzian, Gaussian) have been input into the PDE solver. The analysis technique typically reproduces the input parameters to within 2-3\% accuracy. 


\section{DATA ANALYSIS AND RESULTS}

In order to fit the HXR data to this model, the integrated chordal data is boxcar binned, Fourier decomposed in time, and then spatially inverted to be a function of $r$. Generally speaking, the fundamental and $3^{\text {rd }}$ harmonic are the only Fourier components with enough signal-to-noise to include in the fit. Since the transport terms ( $v$ and D) are small when compared with the source and slowing-down terms, we do the analysis in the radial location where HXR emission is peaked and the first and second derivatives of $\mathrm{n}(\mathrm{r})$ are the largest in order to measure $\mathrm{v}$ and $\mathrm{D}$ as accurately as possible. For the case shown in this paper, the peak HXR emission corresponds to $r$ 0.7. A boxcar binned integrated HXR chord and inverted HXR emissivity example is shown in Fig. 1.
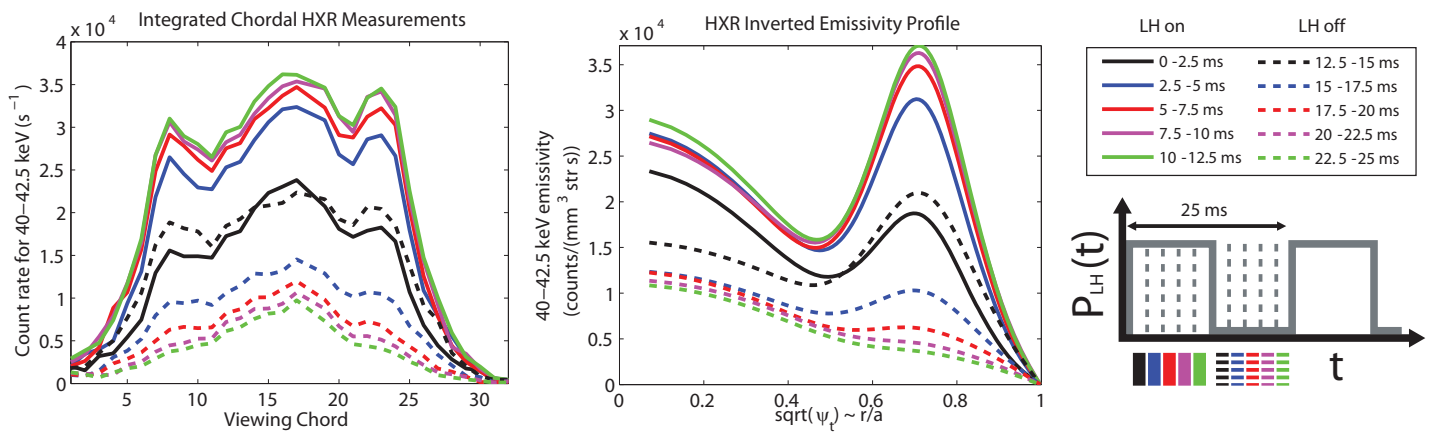

FIGURE 1. Boxcar binned integrated HXR chords and inverted HXR emissivity.

In the local region around the peak HXR emission, various parameters are solved for in the transport model, which fits the right sides of Eq. 3 and Eq. 4 to the left sides of Eq. 3 and Eq. 4. All parameters are solved for with a least-squares approach, with the exception of $\delta t$, which is solved for iteratively. This analysis is performed on each $\mathrm{x}$-ray energy bin separately. Strictly speaking, the various energy bins are coupled, because a slowing-down term in one energy bin is a source term in another. However, we assume they are decoupled for simplicity.

Fig. 2 shows the slowing-down time and pinch velocity recovered from the model, as well as several other parameters solved for in the analysis, as a function of energy. While the dependence of these transport coefficients on x-ray energy is qualitatively suggestive of their dependence on electron energy, it is important to remember that there is not a one-to-one correspondence between photon and electron energy. The diffusion coefficients calculated by the model were less than $5 \times 10^{-3} \mathrm{~m}^{2} / \mathrm{s}$. The diffusion coefficients appeared so small that they may not be accurately measurable in this case. Consequently, diffusion was set to 0 for this scenario. The pinch velocity calculated by the model ranged from 1-2 $\mathrm{m} / \mathrm{s}$, with higher pinch velocities corresponding to higher energy photon bins. 
(a)

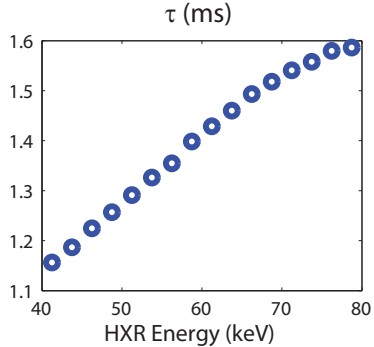

(d)

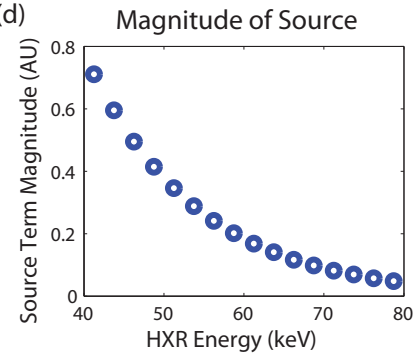

(b)

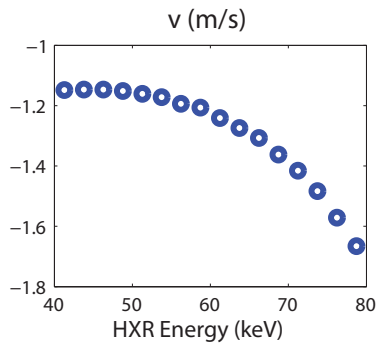

(e)

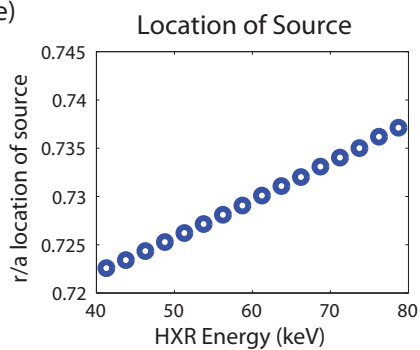

(c)

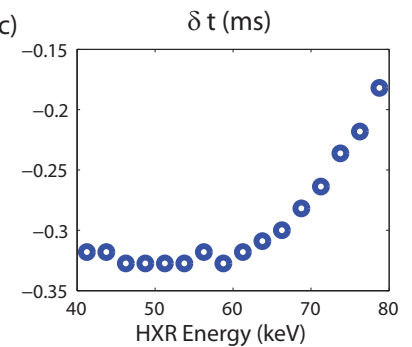

(f)

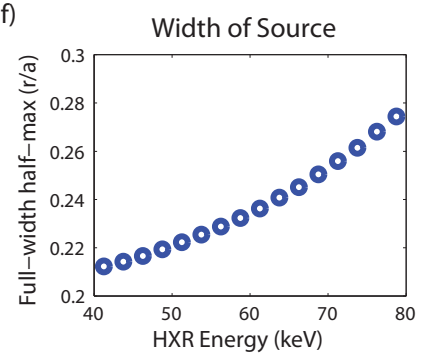

FIGURE 2. Parameters solved for in data analysis. (a) The slowing-down time. Note that this is physically consistent with the relativistic expected slowing-down time and increases with energy as expected. (b) The pinch velocity. A negative velocity indicates the inward direction (a pinch). (c) The time delay between when LH power is turned on and when HXR appears. As expected, lower energies appear first and then the tail builds up, consistent with the theoretically predicted tail build-up time. (d) Magnitude of source term. (e) Radial location of the peak of the source term. (f) Radial width (fullwidth half-max) of the source term.

\section{CONCLUSIONS AND ACKNOWLEDGEMENTS}

In the plasma scenario described in this paper, it appears that the fast electrons are pinching inward with a velocity of $1-2 \mathrm{~m} / \mathrm{s}$. Diffusion appears so small that it is negligible and very difficult to measure accurately. Other parameters that are solved for (slowing-down time, time lag between different energies appearing) appear physically consistent with theoretical results. This analysis technique will be applied to other plasma scenarios for comparison.

The analysis technique has been tested on a model with known parameters and reproduces these parameters well. We could increase confidence in the technique by testing it on time-dependent output from a full-wave solver coupled to a Fokker-Plank code.

Thank you to Craig Petty and Matt Reinke for valuable discussions regarding data analysis techniques. This work is supported by the US DOE awards DE-FC0299ER54512 and DE-AC02-76CH03073.

\section{REFERENCES}

1. S. Knowlton, M. Porkolab, and Y. Takase, Phys. Plasmas, 1, 891-900 (1994).

2. S. Jones, et al, Plasma Phys. and Controlled Fusion, 35, 1003-17, (1993).

3. A. Ince-Cushman, et al, Phys. Rev. Letters, 102, (2009).

4. J. E. Rice, et al, Nuclear Fusion, 49, (2009).

5. J. Liptac, R. Parker, et al, Rev. Sci. Inst., 77, (2006).

6. J. C. Wright, P. T. Bonoli, et al, Phys. Plasmas, 16, to be published (2009). 